\title{
TNF receptor type 1 and TNF receptor type 2 mRNA expression was not associated with coronary artery disease in a group of Iranian Turks
}

\author{
Bagheri $\mathrm{M}^{1,2}$, Khadem-Vatani $\mathrm{K}^{3}$, Seyed-Mohammad-Zad $\mathrm{MH}^{3}$, Abdi Rad I ${ }^{2}$, Rostamzadeh $\mathrm{A}^{3}$, \\ Rahimi B ${ }^{3}$, Mahmoudi-Nejad S ${ }^{1}$
}

School of Medicine, Cellular and Molecular Research Center, Cellular and Molecular Medicine Institute, Urmia University of Medical Sciences, Urmia, Iran. mortezabagheri@umsu.ac.ir

\begin{abstract}
OBJECTIVES: Present investigation was carried out to evaluate the mRNA level of TNF receptor type 1 (TNFR1) and TNF receptor type 2 (TNFR2) in peripheral-blood cells in patients with premature CAD over healthy controls. BACKGROUND: TNFa as a pleiotropic cytokine could be concerned in cardiovascular pathophysiology regarding its special effects on endothelial cells. TNFa exerts its activities through its receptors, TNFR1 and TNFR2. METHODS: Totally, 40 patients with premature CAD and 40 healthy controls were studied. The qRT-PCR technique was used to determine the mRNA level of TNFR1 and TNFR2 in tested groups.

RESULTS: The results of this study show that the relative expression was $1.32 \pm 0.34$ in cases and $1.11 \pm 0.16$ in controls for TNFR1. The relative expression was $0.96 \pm 0.13$ in cases and $1.49 \pm 0.41$ in controls for TNFR2. There is no significant difference in the level of gene expression in the studied groups regarding TNFR1 and TNFR2 genes $(p>0.05)$.

CONCLUSION: It can be concluded that the mRNA levels of TNFR1 and TNFR2 were not associated with CAD risk. Studies with more details, larger sample size, and new risk loci are necessary to reveal disease-causing mechanisms in the pathogenesis of CAD (Fig. 3, Ref. 21). Text in PDF www.elis.sk.

KEY WORDS: gene expression, TNFR1,TNFR2, premature CAD.
\end{abstract}

\section{Introduction}

Cardiovascular disease is the most common cause of death in developing countries (1). The mortality rate from cardiovascular disease is increasing in Iran (2). Approximately one-third of all vascular diseases are related to coronary artery disease (CAD) (3). The traditional risk factors for this disease include lipid disorders, smoking, diabetes, high cholesterol, social and psychosocial factors and stress, lack of daily use of fruits and vegetables, lack of mobility and regular physical activity, family history of CAD and high blood pressure (4). Atherosclerosis is the main reason for the onset of CAD. The incidence of symptomatic CAD in people under 55 years of age, in men and women under 65 years

${ }^{1}$ Student Research Committee, Urmia University of Medical Sciences, Urmia, Iran, ${ }^{2}$ Cellular and Molecular Research Center, Cellular and Molecular Medicine Institute, Urmia University of Medical Sciences, Urmia, Iran, and ${ }^{3}$ Seyyed-al Shohada University Hospital, Urmia University of Medical Sciences, Urmia, Iran

Address for correspondence: M. Bagheri, Dr, School of Medicine, Cellular and Molecular Research Center, Cellular and Molecular Medicine Institute, Urmia University of Medical Sciences, Urmia, Iran.

Phone: +98.44.32770969, Fax: +98.44.32250730

Acknowledgements: This study was funded by Urmia Medical Science University (Grant No: 2062). We are thankful to patients and their families, Seyyed-al Shohada University Hospital staff for providing the samples. of age is called premature CAD (4.5). CAD is primarily caused by the interaction of gene-gene or genetic-environmental factors (6). CAD is a chronic inflammatory disease, and its major cause is arteriosclerosis and the pathological formation of arthroplastic plaques in one or more coronary artery (7). Considering that there is a correlation between the elevation of serum alkaline phosphatase (ALP) and myocardial infarction and coronary death (8), and also because tumor necrosis factor-alpha receptors are the most important factors regulating inflammatory responses; a study of genetic changes and expression of the receptors of the Tumor Necrosis Factor-alpha (TNF $\alpha$ ) in patients with CAD are very valuable (9). According to the above, and also the lack of a similar studies in this field, this study was designed to analyze the expression of TNF receptor type 1 (TNFR1) and TNF receptor type 2 (TNFR2) in peripheral-blood cells in patients with premature CAD.

\section{Materials and methods}

In general, 80 individuals were studied in two groups. The first group included 40 patients with premature CAD (under 50 years old), while the second group consisted of 40 healthy controls. This study was carried out after approving and obtaining necessary permissions from the Urmia University of Medical Sciences's Ethics Committee at the specialized cardiology cen- 

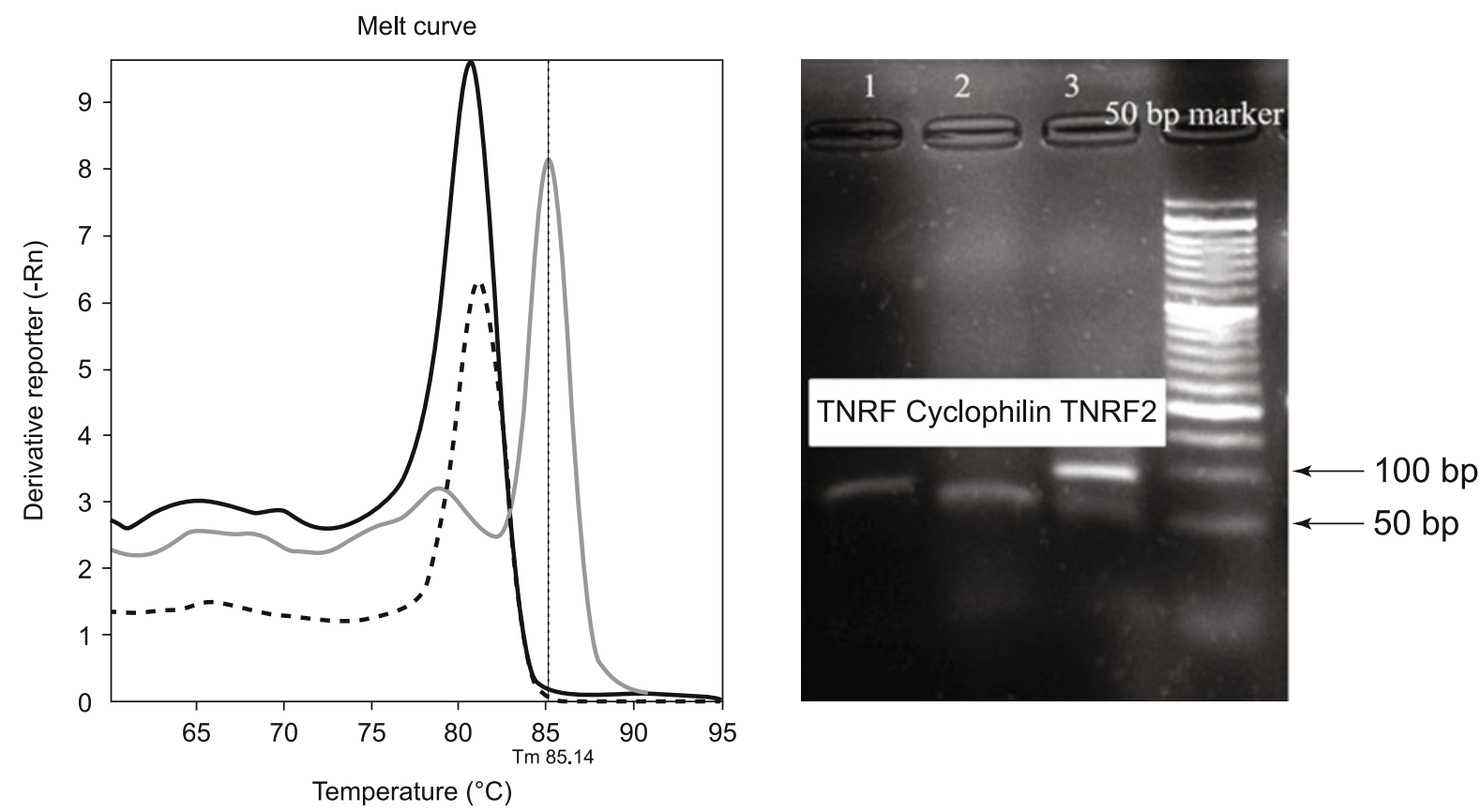

Fig. 1. Melting curve and agarose gel analysis of TNFR1 (76 bp), TNFR2 (99 bp) and Cyclophillin (57 bp) genes on $2.5 \%$ agarose gel in this study.

ter of Sayyid Shohadah and the Cellular and Molecular Medicine Institute, Urmia, Iran. Written consent was obtained from all participants. CAD predisposition less than 50 years old was defined as premature (5). Information about age, sex, family history of CAD, dyslipidemia, diabetes mellitus, hypertension or the use of antihypertensive drugs, cigarette smoking were collected. Clinical,echocardiogram, electrocardiogram and coronary angiography findings were evaluated by an expert cardiac specialists (10). CAD is generally defined as the presence of a more than $50 \%$ stenosis at angiography (11). 2-3 ml blood sample was collected in a Falcon tube containing EDTA. The RNX Plus Solution Kit (SinaClon) (Catalog Number: RN7713C) was used to extract RNA from the specimens according to manufacturer's design with a few changes. Before performing other tests, the quality and purity of the RNA was confirmed. The spectrophotometer (Eppendorf AG, Germany) measured the absorbance of OD of RNA solution and not exceeded by $260 / 280<1.6 O D$. Then, in order to synthesize cDNA, the Thermo Scientific RevertAid First Strand cDNA Synthesis Kit was used in accordance with the manufacturer's guide. The qRTPCR technique was used to determine the level of gene expression in the samples. The sets of primers 5'-cgetaccaacggtggaagtc-3' and 5'-caagctcccctctttttcag-3', 5'-caagccagctccacaatgg-3' and 5'-tgaccgaaaggcacattcct-3', and 5'-tgccatcgccaaggagtag-3' and 5'-tgcacagacggccactcaaa-3' were used for TNFR1 (76 bp), TNFR2 (99 bp) and Cyclophillin (57 bp), respectively. The results of Real time PCR were analyzed using the $2^{-\Delta \Delta C t}$ method (12).

\section{Statistical analysis}

Data analysis was performed using SPSS 20 software. The Kolomogorov-Smirnov test was used to investigate the normal distribution of data. In the case of normal distribution of data, for comparing the mean of data between two groups, t-test for 2-Independent-samples was performed. In the case of non-normally distributed data, for comparing the mean of data between two groups, Mann-Whitney U-test was used for non-parametric variables. A p $<0.05$ is considered significant. The data were reported based on Mean $\pm \mathrm{SE}$.

\section{Results}

The results of this study are summarized in Figures 1 to 3. In this study, 80 patients were studied in two groups of patients (40 patients with an average age of 45 years) and control (40 healthy subjects with an average age of 44 years). The relative expression

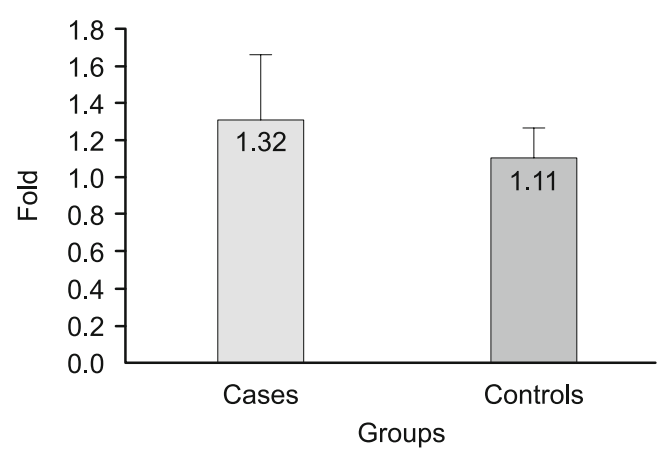

Fig. 2. Mean expression of TNFR1 (fold) in cases and controls. The foldchange of TNFR1 gene in the tested groups was not significant $(p=0.6)$. 


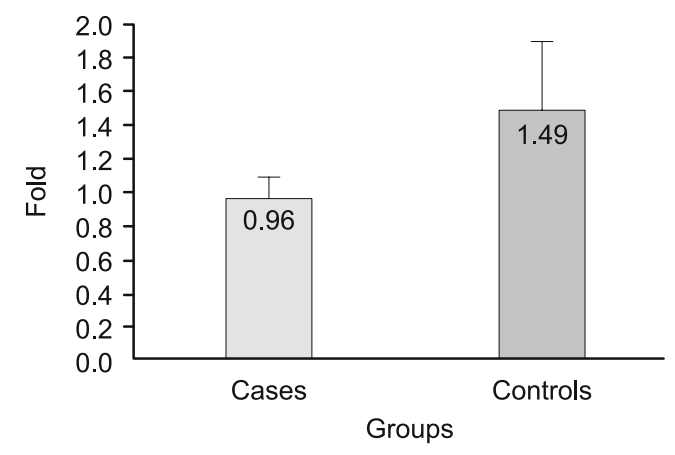

Fig. 3. Mean expression of TNFR2 (fold) in cases and controls. The foldchange of TNFR2 gene in the tested groups was not significant $(p=0.2)$.

was $1.32 \pm 0.34$ in cases and $1.11 \pm 0.16$ in controls for TNFR1. The relative expression was $0.96 \pm 0.13$ in cases and $1.49 \pm 0.41$ in controls for TNFR2. Analysis of the findings of this study shows that there is no significant difference in the level of gene expression in the studied groups regarding TNFR1 and TNFR2 genes $(\mathrm{P}$ - value $>0.05)$. Figure 1 shows melting curve and agarose gel analysis of TNFR1, TNFR2 and Cyclophillin genes in the present examination.

\section{Discussions}

Cardiovascular disease is a public health problem and the main cause of morbidity and mortality in the world (14). TNF $\alpha$ is a strong pleiotropic cytokine that has adverse pro-inflammatory effects. TNF $\alpha$ could be concerned in cardiovascular pathophysiology regarding its special effects on endothelial cells (15). In the vascular system, $\mathrm{TNF} \alpha$ causes a lot of changes in endothelial function and vascular smooth muscle, as well as influences the blood cells. Such changes in vascular dysfunction, the onset and progression of atherosclerosis are very important. TNF $\alpha$ plays a major role in the pathogenesis and progression of atherosclerosis. The production and release of TNF $\alpha$ and its related downstream signaling madiators after activation of its receptors, TNFR1 and TNFR2 have a special effect on the cardiac and vascular systems $(16,17)$. Both are expressed in myocytes of the heart (17). Circulating levels of TNF $\alpha$ can be seen in patients with cardiomyopathy, myocardial infarction and chronic heart failure (18). The findings of several studies indicated controversial results (16-19). HassanNejhad et al (2018) failed to show any association between TNF $\alpha$ gene expression and premature CAD in Iranian Turks. They proposed that other mechanisms in the pathophysiology of premature CAD might be evaluated regarding more deitailes and genes such as TNFR2 (19). In this regard, it has been shown that the ratio of TNF/TNFR can be a predictor of the pathogenesis of the disease (20). In the present study, the expression of TNFR1 and TNFR2 genes was investigated in patients with CAD and healthy controls and the analysis of the findings showed that there are no significant differences in the relative expression (fold-changes). Genetics of CAD is complex. The influence of environmental factors increases the $\mathrm{CAD}$ complexity while traditional risk factors remain vital.
About 60 SNPs with minor allele frequency $>0.05$ have been associated with CAD risk (21). Our study had some limitation regarding low sample size and poor quality of medical records in registry systems. Further studies with more details are necessary to reveal the role of genes and other mediators relevant to vessel wall biology and immune responses.

\section{Conclusion}

It can be concluded that the mRNA levels of TNFR1 and TNFR2 were not associated with CAD risk. Studies with more details, large sample size, gene-gene interactions, and new risk loci are necessary to reveal disease-causing mechanisms in the pathogenesis of CAD.

\section{References}

1. Gaziano TA, Bitton A, Anand Sh, Abrahams-Gessel Sh, Murphy A. Growing Epidemic of Coronary Heart Disease in Low- and Middle-Income Countries. Curr Probl Cardiol 2010; 35 (2): 72-115.

2. Sadeghi M, Haghdoost AA, Bahrampour A, Dehghani M. Modeling the Burden of Cardiovascular Diseases in Iran from 2005 to 2025: The Impact of Demographic Changes. Iran J Public Health 2017; 46 (4): 506-516.

3. Wilson PW, D’Agostino RB, Levy D, Belanger AM, Silbershatz H, Kannel WB. Prediction of coronary heart disease using risk factor categories. Circulation 1998; 18: 1837-1847.

4. Sadeghi R, Adnani N, Erfanifar A, Gachkar L, Maghsoomi Z. Premature coronary heart disease and traditional risk factors-can we do better? Int Cardiovasc Res J 2013; 2: 46-50.

5. Sanchis-Gomar F, Perez-Quilis C, Leischik R, Lucia A. Epidemiology of coronary heart disease and acute coronary syndrome. Ann Transl Med 2016; 4 (13): 256.

6. Pranavchand R, Reddy BM. Current status of understanding of the genetic etiology ofcoronary heart disease. J Postgrad Med 2013; 1: $30-41$.

7. Conti P, Shaik-Dasthagirisaeb Y. Atherosclerosis: a chronic inflammatory disease mediated by mast cells. Cent Eur J Immunol 2015; 40 (3): 380-386.

8. Kunutsor SK, Bakker SJ, Kootstra-Ros JE, Gansevoort RT, Gregson J, Dullaart RP. Serum Alkaline Phosphatase and Risk of Incident Cardiovascular Disease: Interrelationship with High Sensitivity C-Reactive Protein. PLoS One 2015; 10 (7): e0132822.

9. Sbarsi I, Falcone C, Boiocchi C et al. Inflammation and atherosclerosis: the role of TNF and TNF receptors polymorphisms in coronary artery disease. Int J Immunopathol Pharmacol 2007; 20 (1): 145-154.

10. Aggarwal A, Srivastava S, Velmurugan M. Newer perspectives of coronary artery disease in young. World J Cardiol 2016; 8 (12): 728-734.

11. Ha EJ, Kim Y, Cheung JY, Shim SS. Coronary artery disease in asymptomatic young adults: its prevalence according to coronary artery disease risk stratification and the CT characteristics. Korean J Radiol 2010; 11 (4): 425-432.

12. Livak KJ, Schmittgen TD. Analysis of relative gene expression data using real-time quantitative PCR and the 2 (-Delta DeltaC(T)) Method. Methods 2001; 4: 402-408. 
13. Wang P and Heitman J. The cyclophilins. Genome Biol 2005; 6 (7): 226 .

14. Hua XP, Zhang XD, Kwong JS, Zeng XT, Zhang ZJ, Wei WL.Tumor necrosis factor-alpha G-238A polymorphism and coronary artery disease risk: a meta-analysis of 4,222 patients and 4,832 controls. Ther Clin Risk Manag 2015; 11: 1429-1436.

15. Azzawi M, Hasleton P.Tumour necrosis factor alpha and the cardiovascular system: its role in cardiac allograft rejection and heart disease. Cardiovasc Res 1999; 43 (4): 850-859.

16. Natanson C, Eichenholz PW, Danner RL et al. Endotoxin and tumor necrosis factor challenges in dogs simulate the cardiovascular profile of human septic shock.J Exp Med 1989; 169 (3): 823-832.

17. Defer N, Azroyan A, Pecker F, Pavoine C. TNFR1 and TNFR2 signaling interplay in cardiac myocytes. J Biol Chem 2007; 282 (49): 35564-35573.
18. Levine B, Kalman J, Mayer L, Fillit HM, Packer M. Elevated circulating levels of tumor necrosis factor in severe chronic heart failure. $\mathrm{N}$ Engl J Med 1990; 323 (4): 236-241.

19. Hassan-Nejhad M, Bagheri M, Khadem-Vatani K et al. Tumor Necrosis Factor-alpha Gene Expression in PBMCs of Iranian Azeri Turkish Patients with Premature Coronary Artery Disease (Age .50 Years). Maedica (Buchar) 2018; 13 (1): 12-16.

20. Mcdermott MF. TNF and TNFR biology in health and disease. Cell Mol Biol (Noisy-le-grand) 2001; 4: 619-635.

21. McPherson R, Tybjaerg-Hansen A. Genetics of Coronary Artery Disease. Circ Res 2016; 118 (4): 564-578.

Received November 18, 2018. Accepted December 20, 2018. 